The University of San Francisco

USF Scholarship: a digital repository@ Gleeson Library |

Geschke Center

Mathematics

College of Arts and Sciences

2001

\title{
The Breakdown of Synchronization in Systems of Non-identical Chaotic Oscillators: Theory and Experiment
}

Jennifer Chubb

University of San Francisco, jennifer.chubb@gmail.com

Ernest Barreto

Paul So

Bruce J. Gluckman

Follow this and additional works at: http://repository.usfca.edu/math

Part of the Mathematics Commons

\section{Recommended Citation}

Chubb, Jennifer; Barreto, Ernest; So, Paul; and Gluckman, Bruce J., "The Breakdown of Synchronization in Systems of Non-identical Chaotic Oscillators: Theory and Experiment" (2001). Mathematics. Paper 1.

http://repository.usfca.edu/math/1

This Article is brought to you for free and open access by the College of Arts and Sciences at USF Scholarship: a digital repository @ Gleeson Library Geschke Center. It has been accepted for inclusion in Mathematics by an authorized administrator of USF Scholarship: a digital repository @ Gleeson Library| Geschke Center. For more information, please contact repository@usfca.edu. 
International Journal of Bifurcation and Chaos, Vol. 11, No. 10 (2001) 2705-2713

(C) World Scientific Publishing Company

\title{
THE BREAKDOWN OF SYNCHRONIZATION IN SYSTEMS OF NONIDENTICAL CHAOTIC OSCILLATORS: THEORY AND EXPERIMENT
}

\author{
JENNIFER CHUBB*, ERNEST BARRETO*, $\dagger$, PAUL SO and BRUCE J. GLUCKMAN \\ Departments of Mathematics, Physics \& Astronomy, and \\ The Krasnow Institute for Advanced Study, \\ Mail Stop 2A1, George Mason University, \\ Fairfax, VA 22030, USA
}

Received October 17, 2000; Revised January 24, 2001

\begin{abstract}
The synchronization of chaotic systems has received a great deal of attention. However, most of the literature has focused on systems that possess invariant manifolds that persist as the coupling is varied. In this paper, we describe the process whereby synchronization is lost in systems of nonidentical coupled chaotic oscillators without special symmetries. We qualitatively and quantitatively analyze such systems in terms of the evolution of the unstable periodic orbit structure. Our results are illustrated with data from physical experiments.
\end{abstract}

\section{Introduction}

Systems of several interacting nonlinear elements present a very rich variety of behavior. Of particular interest has been the phenomenon of chaos synchronization. Most of the relevant literature has considered coupled systems of identical elements for which the dynamics can be understood in terms of an invariant synchronization manifold. In this paper, we discuss a more general method of analysis of coupled systems and apply it to an experimental system. In particular, we focus on the process of desynchronization, with special emphasis on systems of nonidentical coupled oscillators. We draw particular attention to this case, since it represents almost every experimental situation of interest: in practice, it is very difficult to prepare sets of truly identical oscillators in physical systems. Furthermore, in biological systems, natural oscillators occur with considerable variability. For example, even within each of several different classes of neurons, no two individual neurons are identical. Our methods, which are applicable to experimental data, form the foundation for discussing synchronization in nonidentical coupled chaotic systems in a more general context without making reference to special symmetries or invariant manifolds. We illustrate our results with both numerical calculations and experimental data from electronic circuits.

The synchronization of coupled chaotic oscillators, a phenomenon first noticed many years ago [Fujisaka \& Yamada, 1983] is most conveniently described in terms of a synchronization manifold: when synchronized, the time evolution occurs on a restricted set embedded in the full state space. For systems of coupled identical elements, this synchronization manifold is contained within a plane (or hyperplane) of symmetry and exists for a wide range of coupling. However, for systems that do not possess special symmetries, such as systems of coupled nonidentical elements, this invariant synchronization manifold may become extremely complicated

*JC (experiment) and EB (theory) contributed equally to the authorship of this work.

${ }^{\dagger}$ Author for correspondence. E-mail: ebarreto@gmu.edu 
or even be destroyed as the degree of coupling is decreased.

Previous work has focused on systems of identical coupled elements for which the synchronization manifold $\mathcal{M}$ persists for a large range of coupling and can be easily identified. On $\mathcal{M}$, the individual components evolve identically in time, and are said to exhibit identical synchrony [Pecora \& Carroll, 1990]. As the coupling decreases from a fully synchronized state, a bubbling bifurcation [Ashwin et al., 1994, 1996; Venkataramani et al., 1996a, 1996b] occurs when an orbit within $\mathcal{M}$ (usually of low period [Hunt \& Ott, 1996]) loses transverse stability. In the presence of noise or small asymmetries, a typical trajectory quickly approaches and spends a long time in the vicinity of $\mathcal{M}$, but makes occasional excursions. As the coupling is further decreased, the blowout bifurcation [Ott \& Sommerer, 1994] is observed when $\mathcal{M}$ itself becomes transversely unstable (on average).

The concept of (differentiable) generalized synchrony (GS) [Afraimovich et al., 1986; Rulkov et al., 1995; Kocarev \& Parlitz, 1996; Hunt et al., 1997] extends these ideas. GS relaxes the condition that the state variables evolve identically, and only requires that they be functionally related. However, as the coupling is reduced, this function may become extremely complicated. In particular, if the system lacks special symmetries (as in the case when the coupled elements are not identical), $\mathcal{M}$ may not exist, or its structure may be so complicated that the practical identification of bubbling-type or blowouttype bifurcations is impossible. In this situation, the work described above does not carry over, and a more general description of the desynchronization process beyond the state of generalized synchrony is needed.

We find that the entire desynchronization process can be fruitfully studied by considering the evolution of the system's unstable periodic orbit (UPO) structure as the coupling is varied over a large range [Barreto et al., 2000; So et al., 2000]. Our analysis, discussed in Sec. 3, provides both a qualitative and a quantitative understanding of the desynchronization process, with the advantage of not making reference to invariant manifolds. We introduce in Sec. 2 the numerical and experimental models that have been used in this work, and in Sec. 4 we report the first experimental verification of these theoretical results.

\section{Systems}

We will describe the phenomenology of desynchronization in a general unidirectionally coupled system of nonidentical chaotic maps. ${ }^{1}$ Consider a system of the form:

$$
\left\{\begin{array}{l}
x \rightarrow f(x) \\
y \rightarrow G(x, y ; c) .
\end{array}\right.
$$

Systems such as Eq. (1) are known in the mathematical literature as skew products or extensions. Here we assume that the coupling is such that at $c=1$, the $x$ and $y$ dynamics are in a state of generalized synchrony (i.e. $y=\phi(x)$ ) [Afraimovich et al., 1986; Rulkov et al., 1995; Kocarev \& Parlitz, 1996] and that at $c=0$, the $x$ and $y$ dynamics are completely independent of one another. $f$ and $G$ may be of any dimension. For illustration of our theoretical results, we use in our discussion below the simplest case

$$
G(x, y ; c)=c f(x)+(1-c) g(y)
$$

and take $f$ and $g$ to be quadratic maps with different parameters. (Another simple option is to use dissimilar Hénon maps.) Our arguments are not specific to these choices, and our experimental system is in fact more complicated.

For our experimental system, we constructed two nearly identical circuits $D$ and $R$ based on the generalized Duffing equation

$$
\frac{d^{2} x}{d t^{2}}+\nu \frac{d x}{d t}+N_{L}(x)=A \sin (t)
$$

where $N_{L}(x)$ is a nonlinear term, typically $N_{L}(x)=$ $\left(x^{3}-x\right)$. In our circuits the nonlinear element was constructed with standard resistors and diodes. The response of this nonlinear element is shown in Fig. 1. A nondimensional parameterization of the equations of our circuits is

$$
\frac{d^{2} x}{d t^{2}}+\alpha \frac{d x}{d t}-\left(\beta x^{3}+\gamma x\right)=\delta \sin (t)
$$

where $\alpha=0.124, \beta=0.238$ and $\gamma=1.00$. Each circuit received a common sinusoidal input, the zerophase of which was also used to trigger stroboscopic measurements. In order to break the symmetry, the amplitude $\delta$ of the sinusoidal input to the response

\footnotetext{
${ }^{1}$ It has been shown that unidirectional and bidirectionally coupled systems are locally equivalent. See [Josić, 1998].
} 


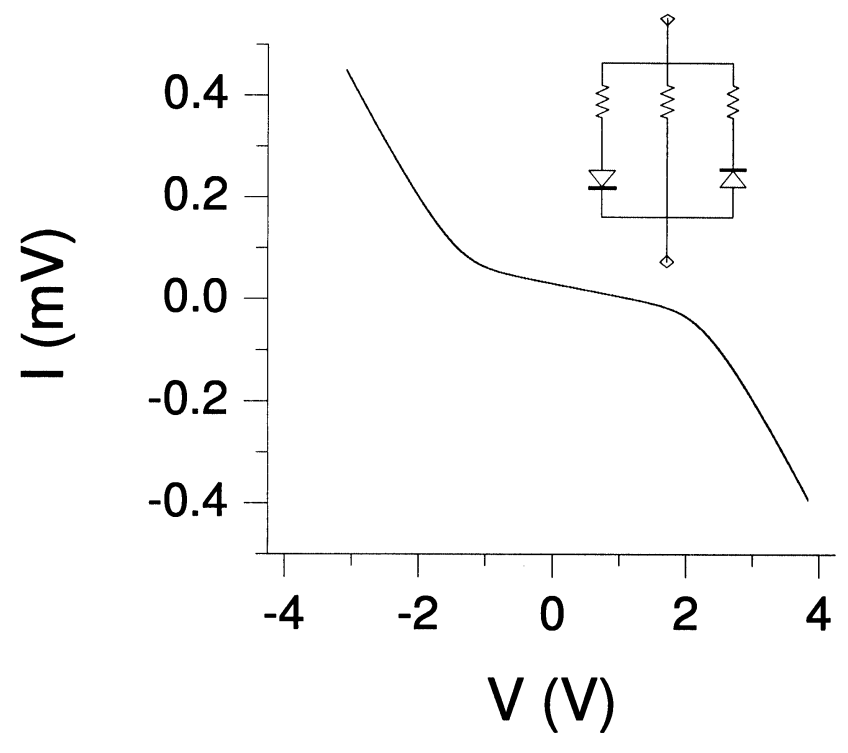

Fig. 1. Current versus voltage curve for the nonlinear element used in our circuits. The same element was used for both the driving and the responding circuits. A polynomial expansion used to fit this curve would include both first- and third-order terms.

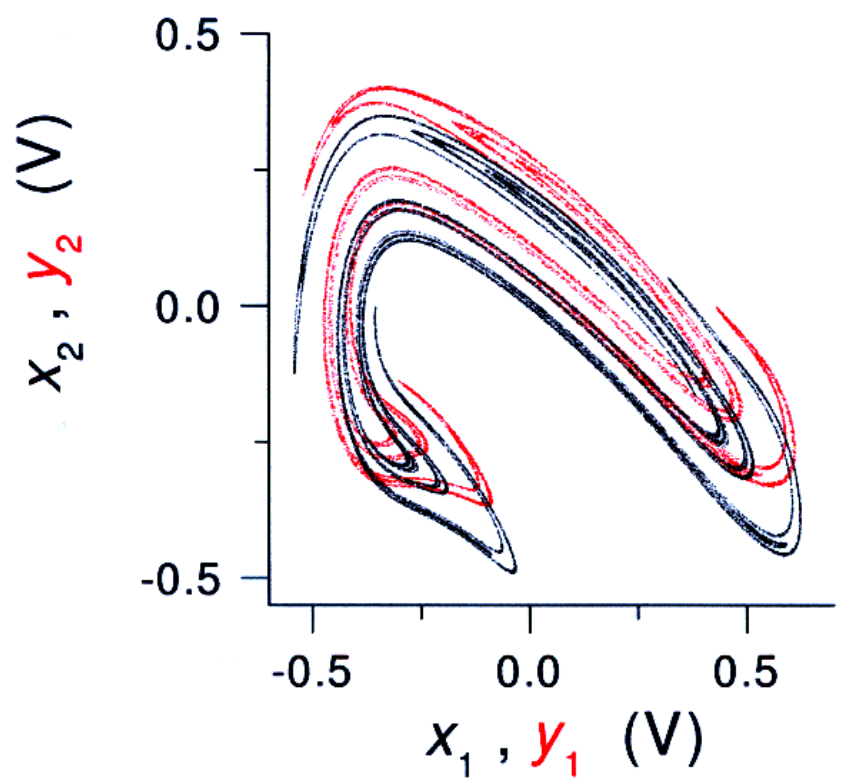

Fig. 2. State space attractors of the driving $(X$, black $)$ and responding $(Y$, red) circuits when uncoupled. Both circuits are driven from the same sinusoidal input but at different amplitudes, resulting in nonidentical attractors. Data was acquired stroboscopically at the zero phase of the sinusoidal input.

circuit $R$ was larger than that used for the driver $D\left(\delta_{R}=17.98\right.$ and $\left.\delta_{D}=13.08\right)$. Accordingly, the attractors are different, as shown in Fig. 2.

The state of each circuit is described by a pair of measurable voltages which we denote by the vec- tors $\mathbf{X}(t)$ and $\mathbf{Y}(t)$; that is, $\mathbf{X}(t)=\left(x_{1}(t), x_{2}(t)\right)^{T}$, with $x_{2} \propto \dot{x_{1}}$, and similarly for $\mathbf{Y}(t)$. The circuits are diffusively coupled together in a driver/response fashion as follows:

$$
\begin{aligned}
& \dot{\mathbf{X}}=D(\mathbf{X}) \\
& \dot{\mathbf{Y}}=R(\mathbf{Y})+d \cdot \mathbf{M}(\mathbf{X}-\mathbf{Y})
\end{aligned}
$$

where $\mathbf{M}$ is a $2 \times 2$ matrix and $d$ is a scalar. For the current work, we use

$$
\mathbf{M}=\left(\begin{array}{ll}
0 & 0 \\
0 & 1
\end{array}\right) .
$$

Identical synchrony occurs for $d \rightarrow \infty$ in this coupling scheme.

The circuits were constructed to be chaotic for relatively low input frequencies $(1-10 \mathrm{~Hz})$, and therefore care was taken to choose low leakage capacitors for the integrator stages and to significantly isolate them from external electrical noise. In addition, the circuits were maintained at constant temperature $( \pm 0.1 \mathrm{C})$, as acquisition runs typically lasted many minutes to hours. Both the coupling and asymmetry are externally voltageprogrammable through the use of a four-quadrant analog multiplier (Analog Devices AD633). Data was acquired with 16-bit precision using a computer acquisition board (National Instruments PCI-MIO16Xe10).

The largest experimentally accessible coupling was $d=9 \mathrm{~V}$, but in this work we only consider a range of $d$ from 1 to $0 \mathrm{~V}$. At $d=0 \mathrm{~V}$, the components oscillate independently. Additional details of this experiment, along with detailed circuit diagrams, will be published elsewhere [Chubb et al., 2001].

\section{Phenomenology of Desynchronization and the Decoherence Transition}

We now describe the process of desynchronization. Our goal is to understand the evolution of the overall periodic orbit structure as the coupling is decreased and synchronization breaks down. Briefly stated: in the absence of special symmetries, the UPOs first undergo a complicated migration apart from one another, after which an important set of new UPOs develops through a series of bifurcations.

In the special case when $f=g$ in Eq. (1b), the synchronization manifold $\mathcal{M}$ is simply the line 
$x=y$. It is invariant and attracting at $c=1$ and remains so until the bubbling bifurcation occurs at a critical value of $c, c_{b u}<1$. In the more general case $f \neq g, x=y$ is by construction invariant and attracting for $c=1$. However, immediately upon decreasing $c, x=y$ is no longer invariant. In Fig. 3, generated numerically with dissimilar quadratic maps for $f$ and $g$, we show the evolution of the attractor of Eq. (1) as the coupling is decreased to zero. Observe that the attractor spreads out as shown in the two magnified views. (This figure is to be compared to Fig. 5 below, which was generated with experimentally measured data.) We first see an apparently multivalued structure appear (top magnification). As the coupling is decreased, we observe a transverse Cantor-like structure in the attractor followed by a "fattening" or "smearing" of the striations as the Lyapunov dimension of the attractor increases to 2.0 (bottom magnification). It is remarkable that this attractor smearing appears to occur well before any embedded UPO loses its transverse stability. In fact, we observe a large range of $c$ over which the periodic orbits migrate apart but do not undergo any bifurcations [Astakhov et al., 1999; Barreto et al., 2000].

The geometry of this migration depends on the invertibility of the driver [So et al., 2000]. If the driver is invertible, this migration of UPOs may result in the loss of differentiability of the (generalized) synchronization manifold [Badii et al., 1988; Pecora \& Carroll, 1996; Hunt et al., 1997; Stark, 1997]. In Fig. 3, the driver is noninvertible.

To continue the description of the desynchronization phenomenology, it is useful to define $U$ to be the set of unstable periodic orbits present on the line $x=y$ when $c=1$ (this definition applies whether or not $f=g$ ). Note that the orbits of $U$ correspond exactly to those of the driver and that their number remains constant for all values of coupling because of the unidirectional coupling. For $f=g$, these UPOs remain fixed in place along $x=y$, but for $f \neq g$, they migrate apart as described above. In both cases, as $c$ is decreased from 1 , the orbits' stability properties evolve, but they remain transversely attracting until a bubbling-type bifurcation is encountered. ${ }^{2}$ This typically corre- sponds to a period-doubling (pitchfork) bifurcation of a low-period orbit in $U$, and leads to the creation of new orbits outside of $U$. As the coupling is further reduced, more and more periodic orbits in $U$ lose their transverse stability in a similar fashion, ${ }^{3}$ leading to the creation of additional orbits. As this process proceeds, the external UPOs simultaneously undergo period-doubling cascades to chaos, thus creating even more new orbits. ${ }^{4}$ We call the set of new orbits created in this fashion the emergent set [Barreto et al., 2000].

The emergent set increases in size and complexity as the coupling is further decreased (see our numerical results in Fig. 3 and experimental results in Fig. 5). This notion can be quantified in terms of topological entropy (for details, see [Barreto et al., 2000]). Intuitively, as more and more UPOs are created outside of $U$, the topological entropy of the emergent set grows, attains a positive value, and continues to increase. At a particular value of coupling, the emergent set's topological entropy equals and then surpasses the topological entropy of the driver. We call this process the decoherence transition. Topological entropy is not an extensive quantity: if $h(A)$ denotes the topological entropy of a set $A$, we have $h(A \cup B)=\max (h(A), h(B))$. Therefore, the decoherence transition corresponds to an abrupt increase in the topological entropy of the entire coupled system.

Although the decoherence transition is not associated with any obvious visual features in the attractor evolution (in Fig. 3 it occurs between the fourth and fifth panels on the left), it does have the advantage of being a quantifiable and experimentally measurable transition which applies to coupled nonidentical chaotic elements. For example, the experimental measurement of the bubbling and/or blowout bifurcation is accomplished by observing excursions from a synchronization manifold whose identification is only practical if $f$ and $g$ are identical, or very nearly so. If $f$ and $g$ are significantly different, the UPO migration described above obscures these bifurcations. The decoherence transition, on the other hand, has the advantage of not requiring the identification of a synchronization manifold for its measurement in practical situations.

\footnotetext{
${ }^{2}$ We extend the concept of bubbling to the asymmetric case $f \neq g$ by defining it as the point where the first orbit in $U$ loses stability.

${ }^{3}$ Additional orbits external to $U$ may be created by saddle-node bifurcation; these may then exchange stability with their corresponding orbits in $U$ via a transcritical bifurcation.

${ }^{4}$ Similar behavior is also seen in bidirectionally coupled systems; see [Astakhov, 1997].
} 


\section{Coupling}

0
0
0
0

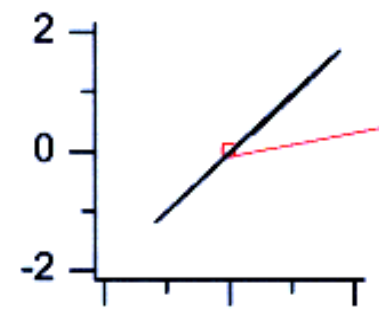

0
0
0
0

\begin{tabular}{l}
0 \\
\multirow{8}{*}{} \\
0 \\
0
\end{tabular}
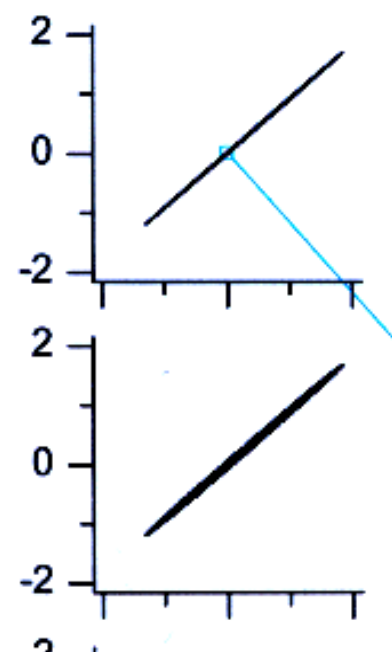

윰

Y
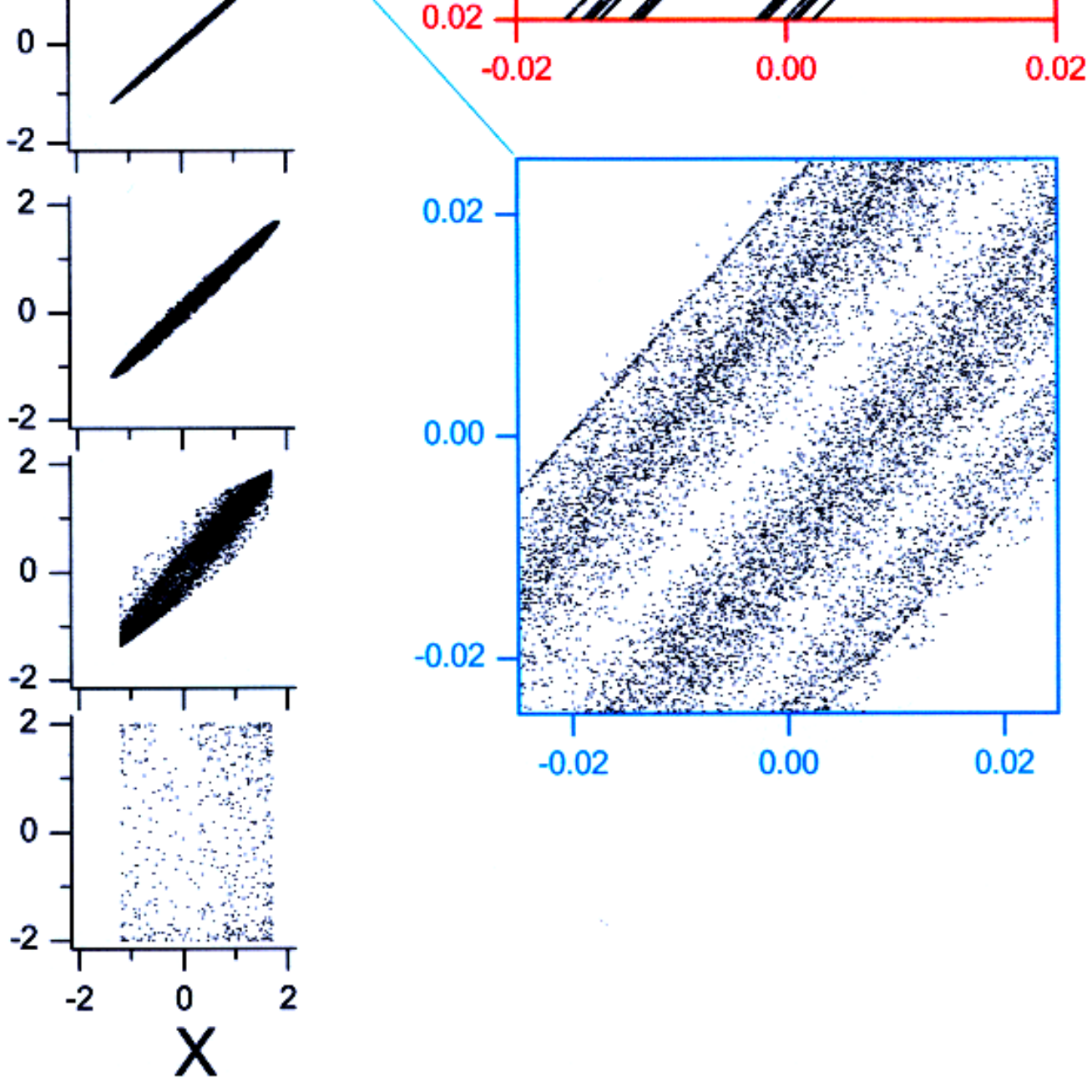

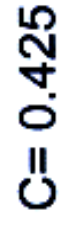

웅

II

II
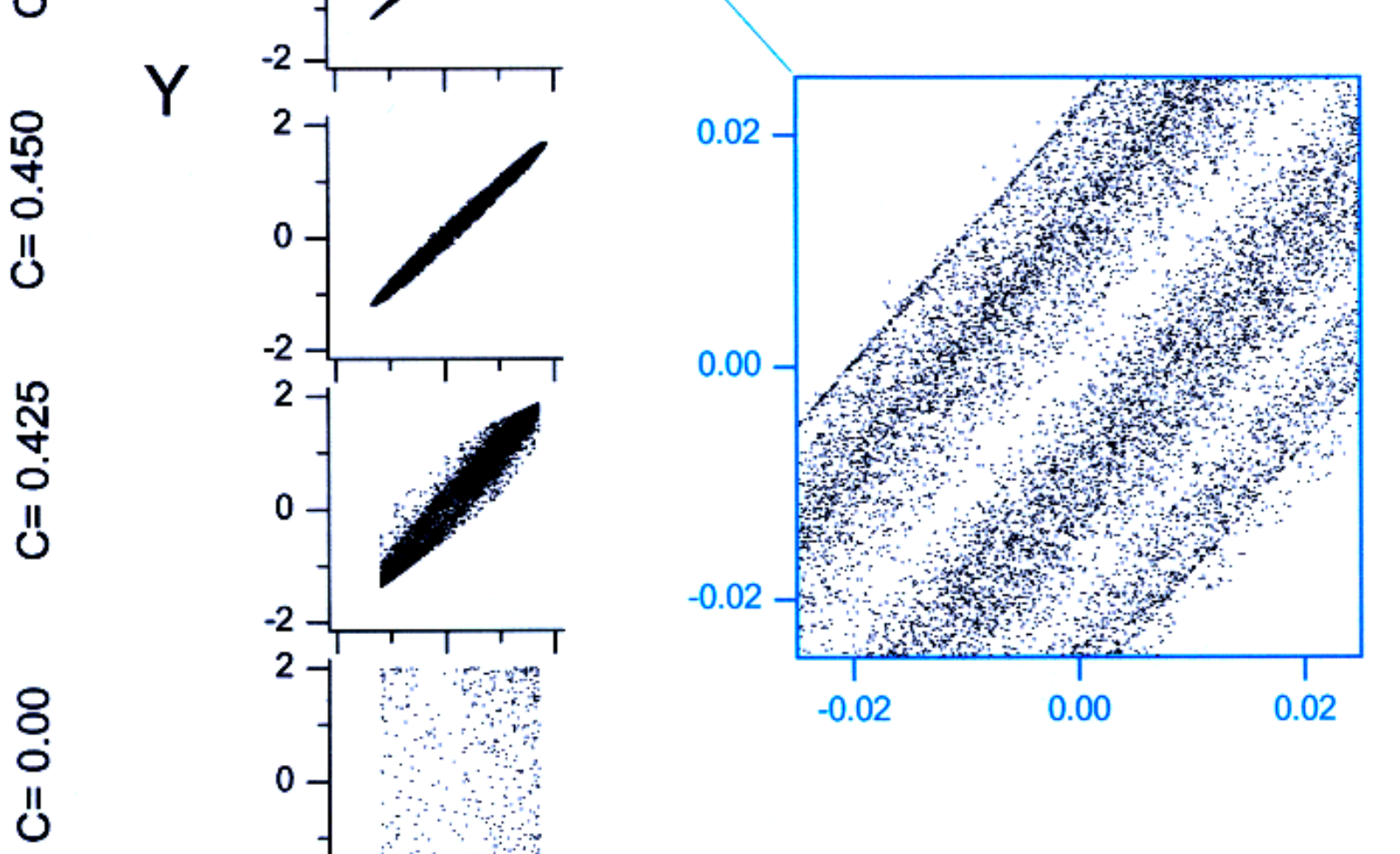

Fig. 3. Attractor evolution as the coupling between elements is decreased to zero for numerical data. Dissimilar quadratic maps $f(x)=1.7-x^{2}, g(y)=2.0-y^{2}$ are used in Eq. (1). The striations and the smearing of the attractor structure described in the text are plainly visible in the magnifications on the right as the coupling $c$ is decreased. The decoherence transition is observed at $c=0.435$. 


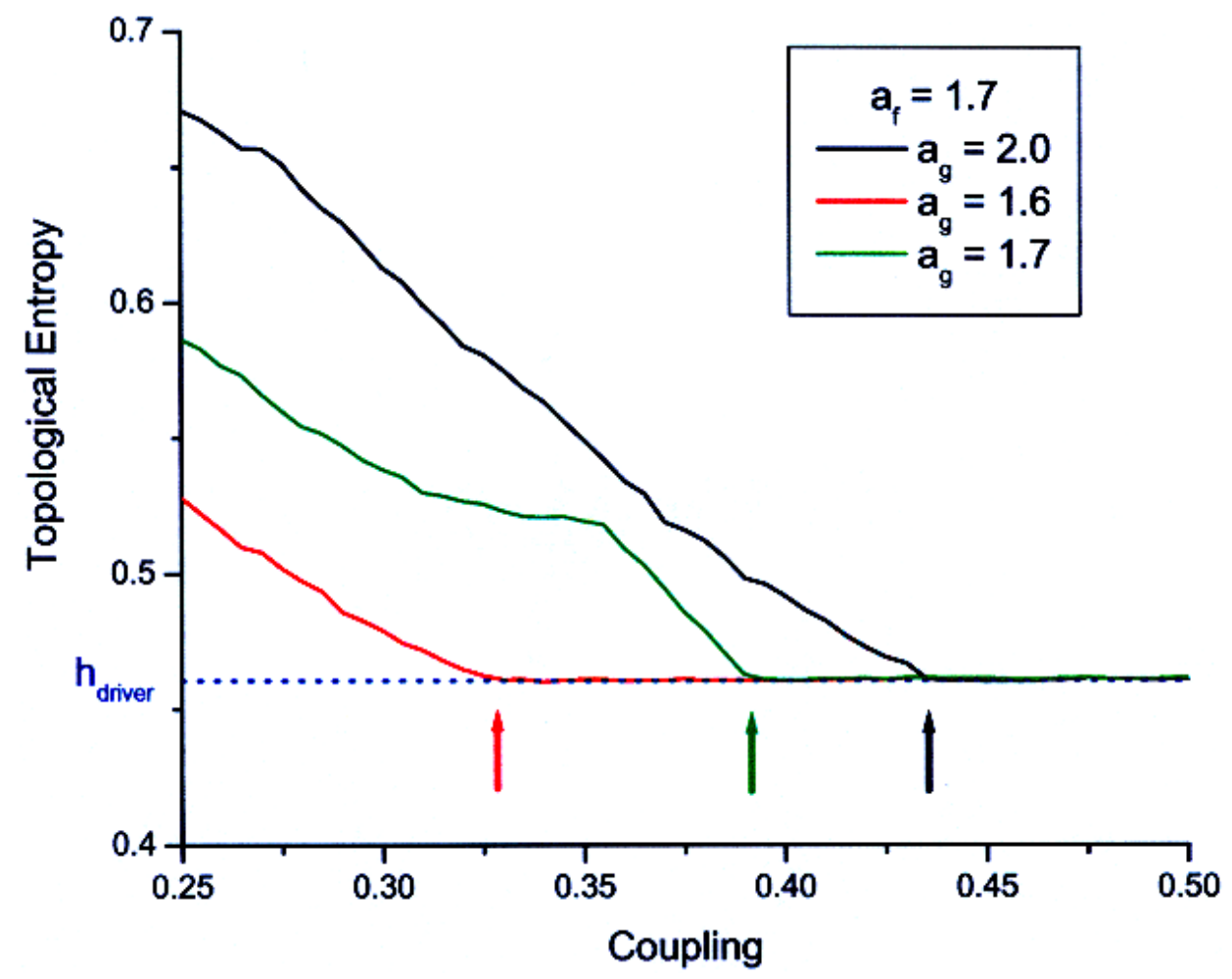

Fig. 4. Numerical results: topological entropy versus coupling for the system in Eq. (1) with $f(x)=1.7-x^{2}, g(y)=a_{g}-y^{2}$, and cases $a_{g}=2.0,1.7$ and 1.6. In all cases the topological entropy is equal to that of the driver (dotted line) for coupling values above the decoherence transition (heavy arrows).

Locating the decoherence transition involves measuring topological entropy from trajectory data. Methods for doing this exist in the literature and involve the measurement of average local expansion rates. An amount of data similar to that needed to obtain reliable Lyapunov exponents is required [Eckmann \& Ruelle, 1996]; for details, we refer the interested reader to [Barreto et al., 2000]. To illustrate the results, we apply these methods to a system of coupled quadratic maps. We take $f(x)=1.7-x^{2}, g(y)=a_{g}-y^{2}$, and consider the cases $a_{g}=2.0,1.7$ and 1.6. Figure 4 shows the topological entropy of the system versus coupling for these cases. In all cases, a clear transition (arrows) is evident where the topological entropy increases abruptly due the increasing complexity of the emergent set.

\section{Experimental Results}

We now report both the experimental observation of the qualitative desynchronization scenario described in Sec. 3 and the quantitative calculation of the decoherence transition from experimental data.
Trajectory data was obtained from coupled electronic circuits as described in Sec. 2.

The process of desynchronization is illustrated in Fig. 5 for experimentally recorded data from our coupled asymmetric generalized Duffing oscillator circuits. As described in Sec. 3, a Cantor-like attractor structure develops as the coupling is decreased from $d=9.00 \mathrm{~V}$ to $d=1.00 \mathrm{~V}$. This structure is shown for $d=1.00 \mathrm{~V}$ in the red magnified view. As $d$ is further decreased, these striations are seen to "fatten" or "smear." The smearing can be seen by comparing the structure in the blue magnification (for $d=0.75 \mathrm{~V}$ ) to that in the red magnification. For successively lower values of the coupling $d$, trajectories are seen to spend less time near the diagonal, thus indicating that the emergent set is developing and becoming more complicated.

The methods referred to above for measuring the decoherence transition can be readily applied to experimentally acquired data. The results for our coupled Duffing circuits are shown in Fig. 6. In order to measure local expansion rates, we estimate the local Jacobians along trajectories from fits of nearby trajectories [Eckmann et al., 1986]. All 


\section{Coupling (V)}

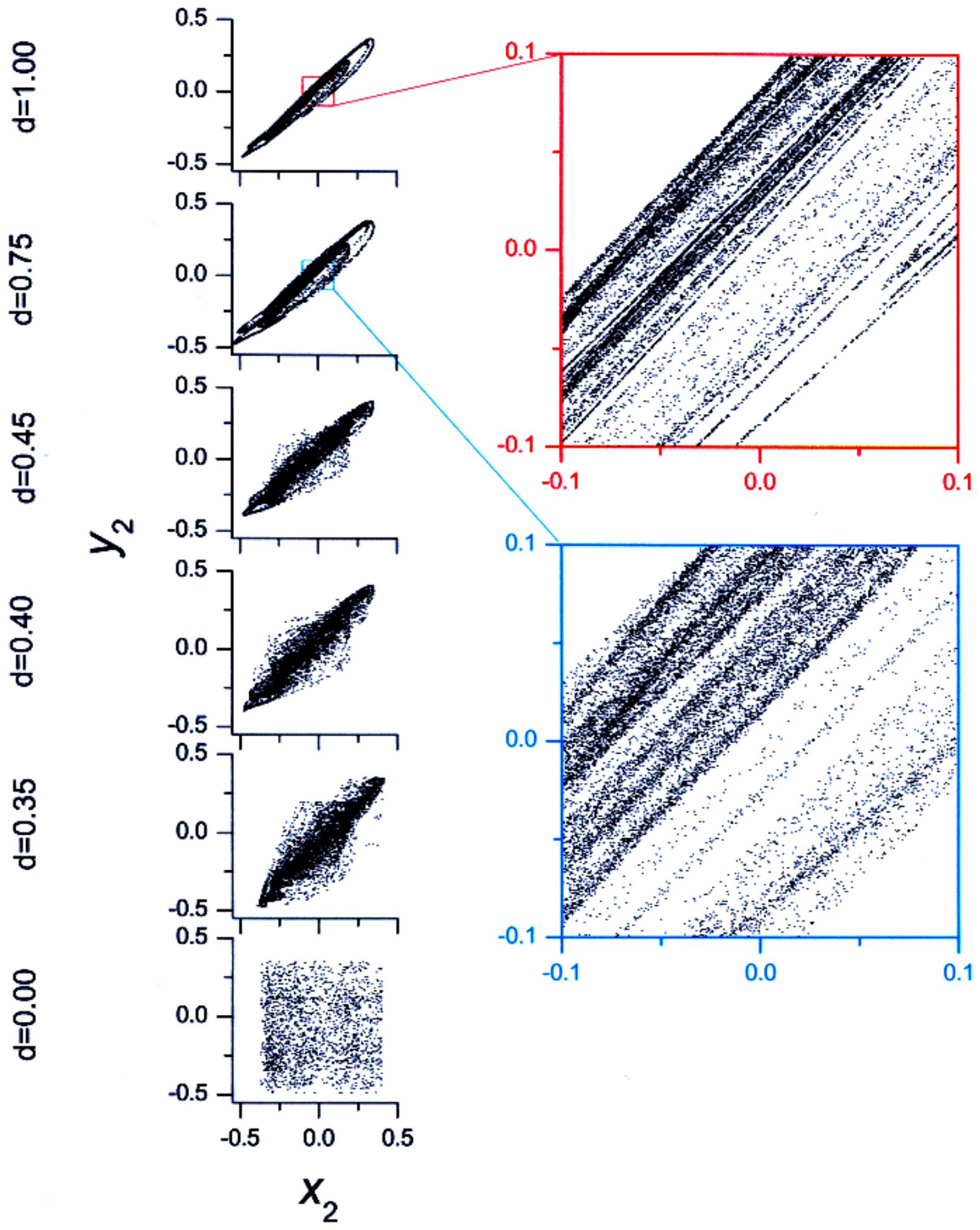

Fig. 5. Attractor evolution as coupling is decreased for diffusively coupled circuits (experimentally measured data). As $d$ is decreased from the largest accessible value, $d=9.00 \mathrm{~V}$, to $d=1.00 \mathrm{~V}$, the attractor develops transverse Cantor-like structure. This is illustrated in the red magnified view of the attractor at $d=1.00$. As coupling is further reduced, this structure becomes smeared, as illustrated in the blue magnified view of the attractor at $d=0.75$. When uncoupled $(d=0)$, there is no correlation between the $X$ and $Y$ dynamics. 


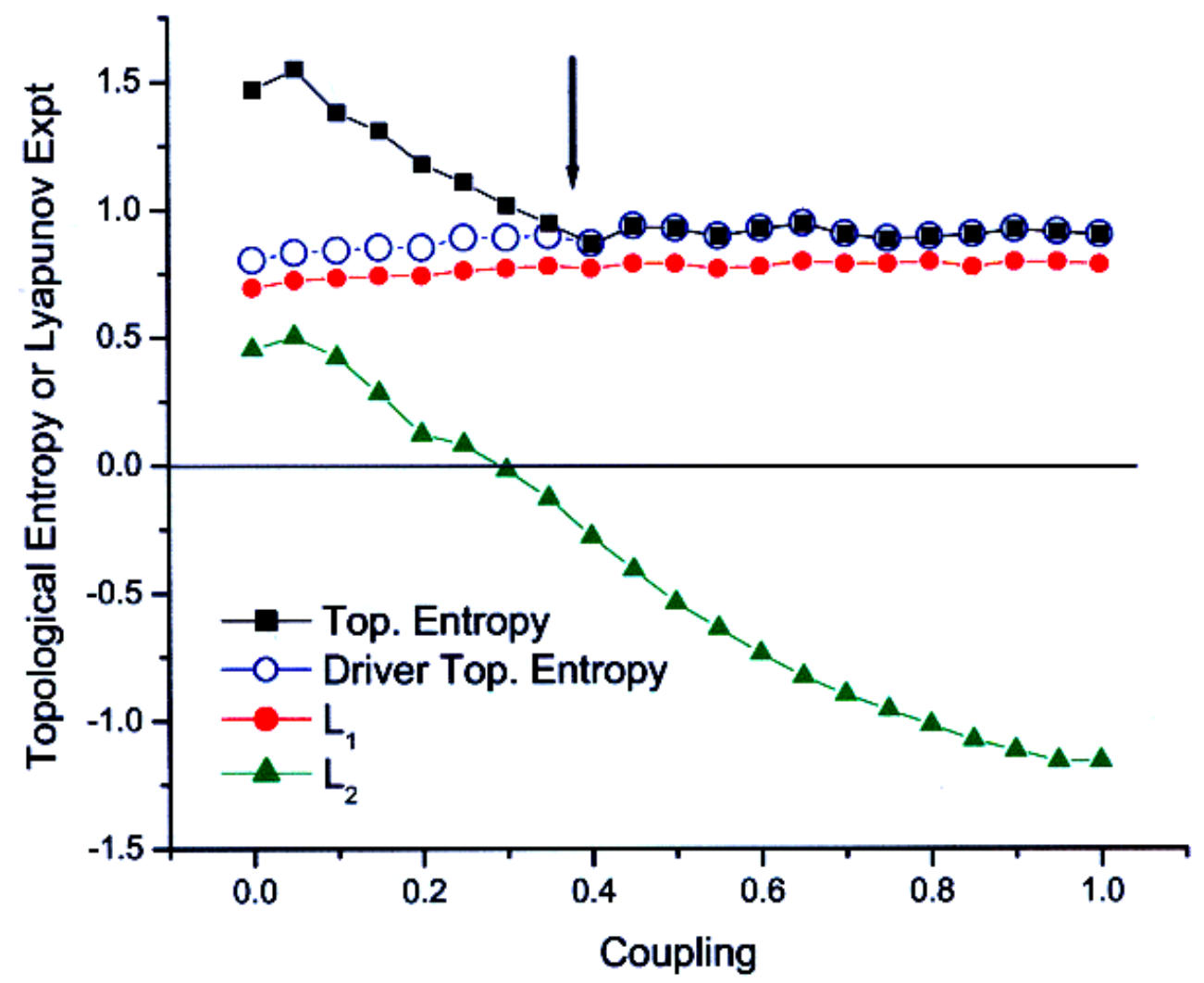

Fig. 6. Experimental results. The topological entropy is plotted as a function of coupling strength. The two largest Lyapunov exponents $L_{1}$ and $L_{2}$ are also plotted for comparison. The decoherence transition occurs when an abrupt increase in the topological entropy is observed (arrow). For this system, the transition occurs between $d=0.35$ and $d=0.40 \mathrm{~V}$.

other steps in the analysis are the same as in the numerical case [Barreto et al., 2000]. We confirm using numerically generated data that the analysis using reconstructed Jacobians yields the same results as when full Jacobian information (from knowledge of the map) is used [Chubb et al., 2001]. We find for our experimental data that the effective decoherence transition occurs at a coupling value within the interval $[0.35,0.40]$, i.e. between the fourth and fifth plots in Fig. 5. Note that there is no obvious visible signature of the decoherence transition in the attractor structure. For comparison, we also plot the two largest Lyapunov exponents versus coupling in Fig. 6.

\section{Conclusion}

The emergent set framework and the subsystem decomposition developed here are quite general and apply to coupled systems of nonidentical elements for which previously studied bifurcation frameworks may be inappropriate. Furthermore, the effective decoherence transition can be estimated for such systems from experimental data, and we report here the first such results.

\section{Acknowledgments}

This work was supported by the National Science Foundation (IBN 9727739) and the National Institutes of Health (2R01MH50006 and 1K25MH01963).

\section{References}

Afraimovich, V. S., Verichev, N. N. \& Ravinovich, M. I. [1986] "Stochastic synchronization of oscillation in dissipative systems," Izv. Vyssh. Uchebn. Zaved. Radiofiz. 29, 1061-1066; Radiophys. Quant. Electron. 29, 795-803.

Ashwin, P., Buescu, J. \& Stewart, I. [1994] "Bubbling of attractors and synchronization of chaotic oscillators," Phys. Lett. A193, 126-139.

Ashwin, P., Buescu, J. \& Stewart I. [1996] "From attractor to chaotic saddle: A tale of transverse instability," Nonlinearity 9, 703-737.

Astakhov, V., Shabunin, A., Kapitaniak, T. \& 
Anishchenko V. [1997] "Loss of chaos synchronization though the sequence of bifurcations of saddle periodic orbits," Phys. Rev. Lett. 79, 1014-1017.

Astakhov, V., Kapitaniak, T., Shabunin, A. \& Anishchenko, V. [1999] "Non-bifurcational mechanism of loss of chaos synchronization in coupled nonidentical systems," Phys. Lett. A258, 99-102.

Badii, R., Broggi, G., Derighetti, B., Ravani, M., Ciliberto, S., Politi, A. \& Rubio, M. A. [1988] "Dimension increase in filtered chaotic signals," Phys. Rev. Lett. 60, 979-982.

Barreto, E., So, P., Gluckman, B. J. \& Schiff, S. J. [2000] "From generalized synchrony to topological decoherence: Emergent sets in coupled chaotic systems," Phys. Rev. Lett. 84, 1689-1692.

Chubb, J., Barreto, E., Schiff, S., So, P. \& Gluckman, B. J. [2001], in preparation.

Eckmann, J.-P., Kamphorst, S. O., Ruelle, D. \& Ciliberto, S. [1986] "Liapunov exponents from time series," Phys. Rev. A34, 4971-4979.

Eckmann, J.-P. \& Ruelle, D. [1992] "Fundamental limitations for estimating dimensions and Lyapunov exponents in dynamical systems," Physica D56, 185-187.

Fujisaka, H. \& Yamada, T. [1983] "Stability theory of synchronized motion in coupled-oscillator systems," Prog. Theor. Phys. 69, 32-47.

Hunt, B. R. \& Ott, E. [1996] "Optimal periodic orbits of chaotic systems," Phys. Rev. Lett. 76, 2254-2257.

Hunt, B. R., Ott, E. \& Yorke, J. A. [1997] "Differentiable generalized synchronization of chaos," Phys. Rev. E55, 4029-4034.
Josić, K. [1998] "Invariant manifolds and synchronization of coupled dynamical systems," Phys. Rev. Lett. 80, 3053-3056.

Kocarev, L. \& Parlitz, U. [1996] "Generalized synchronization, predictability, and equivalence of unidirectionally coupled dynamical systems," Phys. Rev. Lett. 76, 1816-1819.

Ott, E. \& Sommerer, J. A. [1994] "Blowout bifurcations: The occurrence of riddled basins and on-off intermittency," Phys. Lett. A188, 39-47.

Pecora, L. M. \& Carroll, T. L. [1990] "Synchronization in chaotic systems," Phys. Rev. Lett. 64, 821-824.

Pecora, L. \& Carroll, T. [1996] "Discontinuous and nondifferential functions and dimension increase induced by filtering chaotic data," Chaos 6, 432-439.

Rulkov, N. F., Sushchik, M. M., Tsimring, L. S. \& Abarbanel, H. D. I. [1995] "Generalized synchronization of chaos in directionally coupled chaotic systems," Phys. Rev. E51, 980-994.

So, P., Barreto, E., Josić, K., Sander, E. \& Schiff, S. J. [2000], submitted.

Stark, J. [1997] "Invariant graphs for forced systems," Physica D109, 163-179.

Venkataramani, S. C., Hunt, B. R., Ott, E., Gauthier, D. J. \& Bienfang, J. C. [1996a] "Transitions to bubbling of chaotic systems," Phys. Rev. Lett. 77, 5361-5364.

Venkataramani, S. C., Hunt, B. R. \& Ott, E. [1996b] "Bubbling transition," Phys. Rev. E54, 1346-1360. 